\title{
Finite Element Modeling of Propellant and Inhibitor Interface Debonding
}

\author{
Yu Jia-quan, Zheng Jian, Jia Deng \& Zhou Chang-sheng \\ School of Mechanical Engineering, Nanjing University of Science and Technology, Nanjing, \\ 210094, China
}

Keywords: Solid rocket motor, Interface debonding, Virtual crack closure technique, Cohesive zone model, Finite element method.

\begin{abstract}
Interface debonding is one of the most commonly observed failure in the Solid Rocket Motor(SRM). In order to investigate the debonding properties, the experiment was conducted by using double cantilever beam(DCB) specimen, and the strain energy release rate was obtained based on the load-displacement curve. Besides, two finite element methods, virtual crack closure technique(VCCT) and cohesive zone model(CZM), were employed to model the DCB debonding process. Finally, the load-displacement simulation curves obtained based on these models were compared with the experiment curves. The result shows that CZM can model the crack onset and propagation well. And, the VCCT approach, can model the propagation process, but overestimate the initial slope and load peak.
\end{abstract}

\section{Introduction}

Interface debonding is one of the most common observed failure models in the Solid Rocket Motor(SRM)[1]. Bonding interface strength between the propellant and inhibitor is usually lower than strength of the adherends. During the process of manufacturing, storing, transportation and launching, the debonding crack may onset and propagate undetected under temperature shock, fatigue and creep loads. This failure of debonding leads to the burning surface and combustor pressure increasing rapidly, which may cause the ultimate failure of the Solid Rocket Motor[2][3]. According to the statistics, about one-third failure of the SRM is caused by interface debonding. So, the research of the bonding property between the propellant and inhibitor is quite important.

\section{Experiment}

\subsection{Specimen preparing}

In order to acquire the strain energy release rate of the bonding interface between the propellant and inhibitor, double cantilever beam (DCB) specimens were produced according to the ISO 15024:2001(E)[4]. As shown in Fig.1. The specimen consists of aluminum plates, inhibitor, propellant and adhesive, some major dimensions were given in Fig.1.

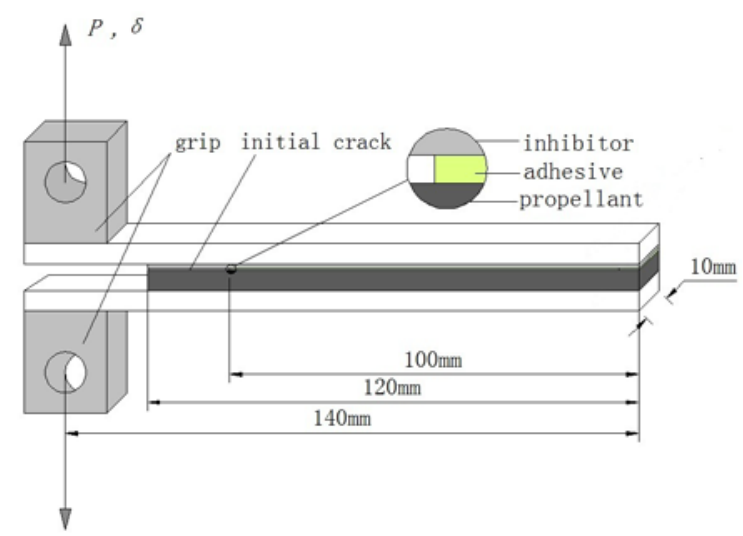

Fig.1: Double cantilever beam (DCB) specimen 
Every surface was cleaned up by using acetone prior to bonding. A kind of high bonding strength adhesive was used on the Inhibitor/Al and propellant/Al interfaces, to make sure the surface don't debond during the experiment process. The inhibitor and propellant were attached to each other using an polyurethane adhesive, which had been proved to have a good bonding effect between the inhibitor and propellant in the SRM. The thickness of the adhesive layer was $0.2 \mathrm{~mm}$, and was controlled by a few steel balls. A $0.2 \mathrm{~mm}$ thickness teflon film was inserted into the adhesive layer to produce the initial crack, the length of the initial crack was $40 \mathrm{~mm}$. All the specimens were stored in the temperature control box at $50^{\circ} \mathrm{C}$ for $48 \mathrm{~h}$ before testing.

\subsection{Testing process and result}

All the specimens were tested on a tensile testing machine at a loading speed of $\mathrm{V}=1 \mathrm{~mm} / \mathrm{min}$ shown in Fig.2. During the experiment process, the location of crack tip was detected by an electron microscope, the length of the crack was determined by the scale which was attached to the aluminum plate. The experiment load-displacement curves and the average curve were given in Fig.3.The curves consist of the linear ascending stage and softening descending stage. As the length of the crack was constant before the crack propagated, the $P-\delta$ curve showed a linear ascending performance. The force decreased when the load peak was reached, which means that the crack was going to propagation.
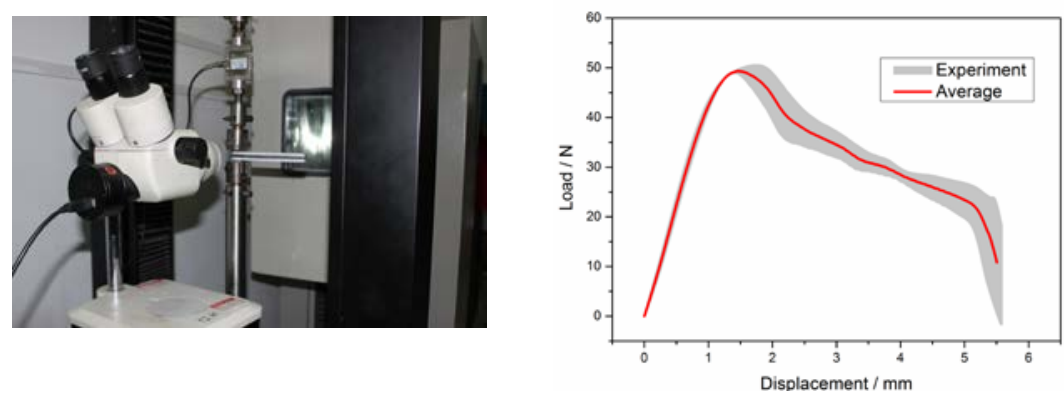

Fig.2: Testing system Fig.3: Experiment load-displacement curves

The strain energy release rate can be obtained by the following equation derived from correction beam theory:

$$
G=\frac{3 P \delta}{2 b(a+|\Delta|)} \times \frac{F}{N}
$$

Using equation (1), the strain energy release rate of this DCB test under $V=1 \mathrm{~mm} / \mathrm{min}$ is $G=0.17 \mathrm{~kJ} / \mathrm{m}^{2}$.

\section{Numerical method}

\subsection{Virtual crack closure technique (VCCT)}

The virtual crack closure technique (VCCT) is an effective method in solving delamination problems. It was first proposed by Rybicki and Kanninen [5] based on the principles of Linear elastic fracture mechanics(LEFM), and widely used in modelling delamination growth in composites[6].

VCCT is based on the assumption that the strain energy released in the crack propagation process is equal to the energy required to close the crack to its original state. The critical strain energy release rate and the crack propagation path should be given when the VCCT theory applied. In this method, the crack path is modeled using pairs of coincident nodes, and two steps are needed to obtain the strain energy release rate(SERR). First step, the node forces at the crack tip before crack propagation are confirmed. Second step, crack tip nodes are released when the load increases to the critical value, and the corresponding node displacements are acquired. SERR is equal to the work during this two steps, which is obtained by multiplying the corresponding node 
displacements with one-half of the node forces.

There is no mode III debonding in the DCB test. So using a 2D VCCT model (Fig.4a) for the DCB crack propagation research, the SEGG can be formulated as:

$$
\begin{aligned}
G_{I} & =\frac{Y_{1}}{2 \Delta a}\left(v_{2}-v_{2^{\prime}}\right) \\
G_{\text {II }} & =\frac{X_{1}}{2 \Delta a}\left(u_{2}-u_{2^{\prime}}\right)
\end{aligned}
$$

where:

$X_{1}$ : horizontal nodal force at crack tip

$Y_{1}$ : vertical nodal force at crack tip

$\Delta a$ : crack length increment

$u_{2}, u_{2^{\prime}}$ : horizontal nodal displacements at crack tip

$v_{2}, v_{2^{\prime}}$ : vertical nodal displacements at crack tip

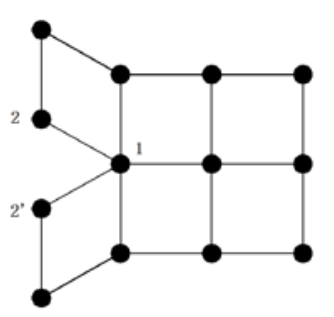

(a)

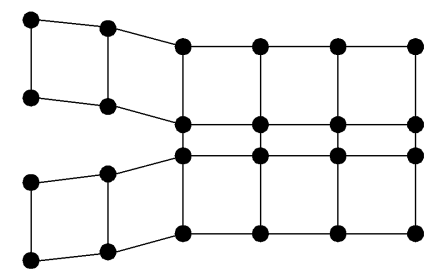

(b)

Fig.4: 2D VCCT model and CZM model

\subsection{Cohesive zone model (CZM)}

Cohesive zone model approach is another efficient method to simulation the interface debonding which was proposed by Dugdale[7] and Barenblatt[8]. The CZM finite element model can be described as Fig4(b), cohesive elements are embed between the adherends to characterize the interface debonding property.

So far, several types of CZM models have been proposed, they can be distinguished by their own cohesive laws[9]. A typical bilinear cohesive law is given in Pic.5, it relates cohesive surface traction, ${ }^{t}$, to displacement jump, $\delta$, at the interface where a debonding may occur. The interface begins to damage when the maximum traction ${ }^{T_{c}}$ reached, and complete failure occurs at the maximum displacement $\delta_{\mathrm{f}}$. The area under the traction-displacement curve is equal to the fracture toughness $G$, which has the same value with SERR. The slope of the traction-displacement curve before damage initial, $\mathrm{K}$, is refereed as the interface stiffness. $\mathrm{K}, T_{\mathrm{c}}$ and $\mathrm{G}$ are the basic parameters to confirm a bilinear CZM model.

The traction-displacement relation can be expressed by:

$$
t=\left\{\begin{array}{cl}
\frac{T_{\mathrm{c}}}{\delta_{0}} \delta & \left(\delta \leq \delta_{0}\right) \\
(1-D) \frac{T_{\mathrm{c}}}{\delta_{0}} \delta & \left(\delta>\delta_{0}\right)
\end{array}\right.
$$

where, $\mathrm{D}$ is damage factor, can be expressed by:

$$
D=\left\{\begin{array}{cc}
0 & \left(\delta \leq \delta_{0}\right) \\
\frac{\delta_{\mathrm{f}}\left(\delta-\delta_{0}\right)}{\delta\left(\delta_{\mathrm{f}}-\delta_{0}\right)} & \left(\delta>\delta_{0}\right)
\end{array}\right.
$$




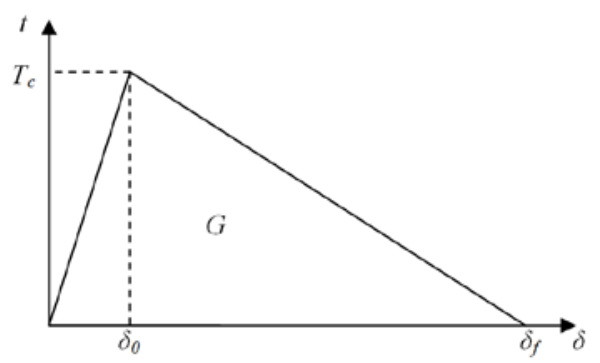

Fig.5: The traction-separation curve of bilinear cohesive zone model

\section{Finite element model simulation analysis}

In order to investigate the propellant/inhibitor interface debonding property by the VCCT approach and CZM approach. The models were composed by using the commercial finite element software ABAQUS in Fig.6. In the VCCT model, the interface between two adherends was defined by the VCCT theory. And a four-node 2D cohesive element (COH2D4) was used to characterize the interface property in the CZM model. The aluminum plates, inhibitor and propellant were modeled with four-node 2D quadrilateral solid element (CPS4R). The VCCT interface parameters are listed in the Table 1, and the CZM cohesive parameters are listed in the Table 2, the maximum normal stress and interface stiffness have been obtained in prior research.

Some researches show that the mess size has obviously effect on the result in the VCCT finite element model analysis. Using a smaller mesh may obtain a better result, but it costs more computing time. Besides, the stress oscillation singularity occurs when the mess size is smaller than a limit value[10]. In order to obtain a relative accurate result and reduce the computing time, $2 \mathrm{~mm}$ length mesh was chosen in the VCCT model.

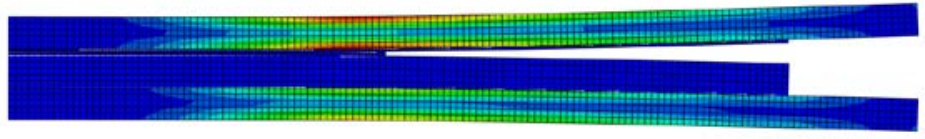

Fig.6: 2D DCB test Finite element model

Table 1: Interface property parameter for VCCT VCCT (Interface property)

Fracture toughness for $\quad 0.17 \mathrm{~kJ} / \mathrm{m}^{2}$ mode I

Table 2: Cohesive element parameters for CZM

\begin{tabular}{cc}
\hline \multicolumn{2}{c}{ CZM (Cohesive element property) } \\
\hline $\begin{array}{c}\text { Fracture toughness for } \\
\text { mode I }\end{array}$ & $0.17 \mathrm{~kJ} / \mathrm{m}^{2}$ \\
\hline Maximum normal stress & $1.5 \mathrm{MPa}$ \\
\hline Interface stiffness & $8 \mathrm{~N} / \mathrm{mm}^{3}$ \\
\hline
\end{tabular}

The simulation load-displacement curves and the experiment curve were given in the Fig.7. The CZM approach can describe the onset and propagation process well in the mode I debonding. The VCCT approach can model the propagation process, but it overestimates the curve's initial slope and load peak. This phenomenon can be explained by the influence of different constitutive between the two theories. In the VCCT theory, the node force increases all the time without a decreasing stage. So, the interface stiffness is larger than initial stiffness of the cohesive element under the same strain energy release rate, which leads to the over prediction of the initial slope and load peak.

Besides, the VCCT simulation load-displacement curve is rough and choppy during the 
propagation process, but still follows the same trend as experiment result. The reason for this phenomenon attributes to the inappropriate mesh size.

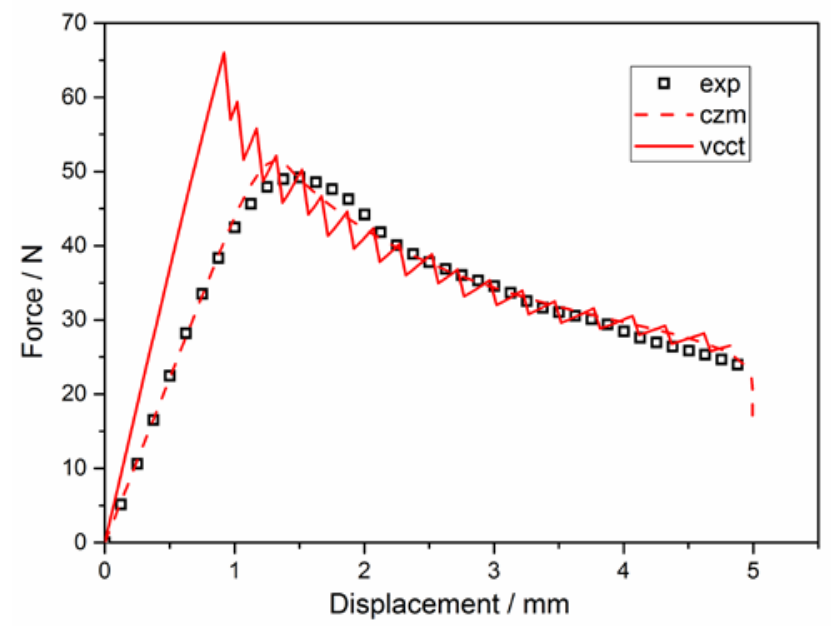

Fig.7: Comparisons between experiment curve and simulation curves

\section{Conclusion}

In this paper, the interface debonding property between inhibitor and propellant in the solid rocket motor was studied. And the experiment was conducted by using double cantilever beam(DCB) specimen to obtain the strain energy release rate(SERR). The VCCT approach and CZM approach were introduced to simulate the debonding process. The result shows that CZM can describe the crack onset and propagation well. But, VCCT will overestimate the initial slope and load peak. Besides, VCCT shows obvious dependency on the mesh size, which means it is important to choose an appropriate mesh.

For CZM modeling, three important parameters (K, $T_{c}$ and $\mathrm{G}$ ) are necessary, and more relevant research should be conducted to obtain the parameters, the prediction for interface debonding is more precision. However, only the strain energy release rate(SERR) is needed for VCCT modeling, which can be achieved by the DCB experiment. So, VCCT is recommended for the primary research.

\section{Acknowledgements}

The research work was supported by Nanjing University of Science and Technology. The authors would like to thank Sun Chao-xiang for proof reading the paper. And the corresponding author is Dr Zheng Jian.

\section{References}

[1] Chang I S. Investigation of space launch vehicle catastrophic failures. Journal of spacecraft and rockets, 33(2), pp.198-205,1996.

[2] Byrd J D, Field L. Method of case bonding propellant: U.S. Patent 4,337,218[P]. 1982-6-29.

[3] Zhou Q C, Ju Y T, Wei Z, et al. Cohesive zone modeling of propellant and insulation interface debonding. The Journal of Adhesion, 90(3), pp.230-251,2014.

[4] ISO15024:2001(E), Fibre-reinforced plastic composites-Determination of mode I interlaminar fracture toughness, $\mathrm{G}_{\mathrm{IC}}$, for unidirectionally reinforced materials.

[5] Rybicki E F, Kanninen M F. A finite element calculation of stress intensity factors by a modified crack closure integral. Engineering Fracture Mechanics, 9(4), pp.931-938,1977.

[6] Shen F, Lee K H, Tay T E. Modeling delamination growth in laminated composites. Composites Science and Technology, 61(9), pp.1239-1251,2001. 
[7] Dugdale D S. Yielding of steel sheets containing slits. Journal of the Mechanics and Physics of Solids, 8(2), pp.100-104,1960.

[8] Barenblatt G I. The formation of equilibrium cracks during brittle fracture. General ideas and hypotheses. Axially-symmetric cracks. Journal of Applied Mathematics and Mechanics, 23(3), pp.622-636,1959.

[9] Chandra N, Li H, Shet C, et al. Some issues in the application of cohesive zone models for metal-ceramic interfaces. International Journal of Solids and Structures, 39(10), pp.28272855,2002.

[10] LU Guo-fu, LIU Yong, ZHANG Cheng-lin. Analysis of strain energy release rate based on virtual crack closure technique. Acta Materiae Compositae Sinica, 26(2), pp.210-216,2009. 